\title{
Rational use of SARS-CoV-2 polymerase chain reaction tests within institutions caring for the vulnerable [version 1; peer
}

\section{review: 2 approved]}

\author{
Tom A. Yates (iD)1, Graham S. Cooke1, Peter MacPherson (iD) 2-4 \\ ${ }^{1}$ Department of Infectious Disease, Faculty of Medicine, Imperial College London, St Mary's Campus, Norfolk Place, London, W2 \\ 1PG, UK \\ ${ }^{2}$ Malawi-Liverpool-Wellcome Trust Clinical Research Programme, Queen Elizabeth Central Hospital, Blantyre, Malawi \\ ${ }^{3}$ Department of Clinical Sciences, Liverpool School of Tropical Medicine, Pembroke Place, Liverpool, L3 5QA, UK \\ ${ }^{4}$ Clinical Research Department, London School of Hygiene \& Tropical Medicine, Keppel Street, London, WC1E 7HT, UK
}

V1 First published: 02 Jul 2020, 9:671

https://doi.org/10.12688/f1000research.24872.1

Latest published: $02 \mathrm{Jul}$ 2020, 9:671

https://doi.org/10.12688/f1000research.24872.1

\section{Open Peer Review}

Approval Status
Persion 1
Institute, Kilifi, Kenya
2. Sheila Lumi University, Kilifi, Kenya
UK University of Oxford, Oxford,
Oxford University Hospital NHS Foundation
Trust, Oxford, UK
Katie Jeffery, Oxford University Hospitals
NHS Foundation Trust, Oxford, UK
Any reports and responses or comments on the
article can be found at the end of the article.

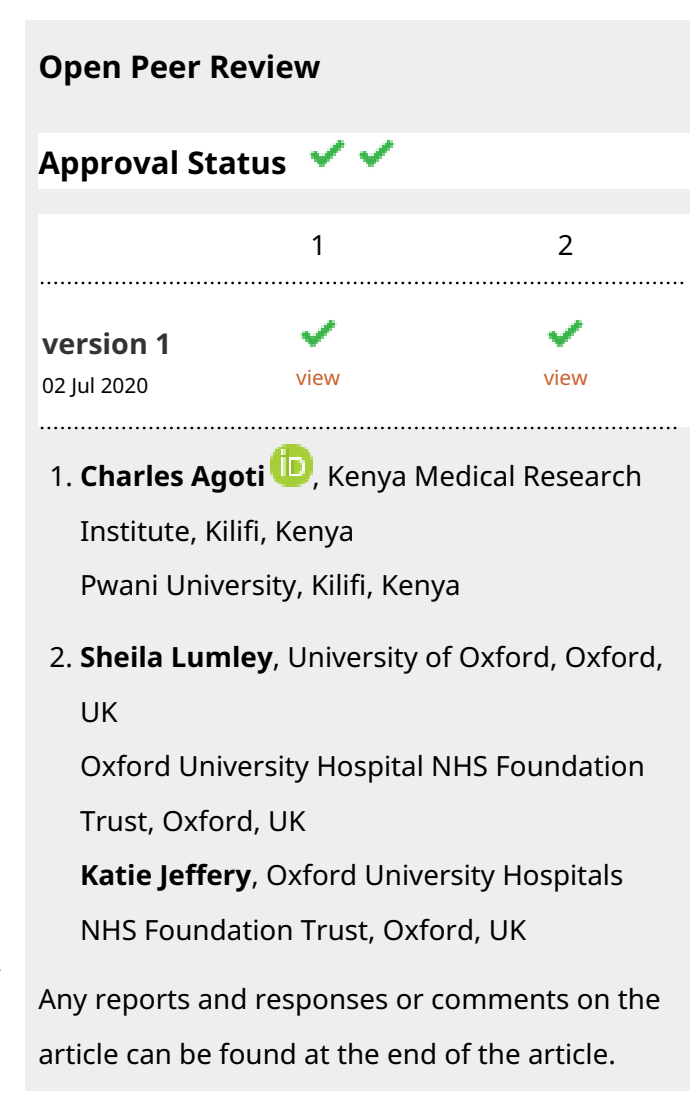

\begin{abstract}
Institutions such as hospitals and nursing or long-stay residential homes accommodate individuals at considerable risk of mortality should they acquire SARS-CoV-2 infection. In these settings, polymerase chain reaction tests play a central role in infection prevention and control. Here, we argue that both false negative and false positive tests are possible and that careful consideration of the prior probability of infection and of test characteristics are needed to prevent harm. We outline evidence suggesting that regular systematic testing of asymptomatic and pre-symptomatic individuals could play an important role in reducing transmission of SARS-CoV-2 within institutions. We discuss how such a programme might be organised, arguing that frequent testing and rapid reporting of results are particularly important. We highlight studies demonstrating that polymerase chain reaction testing of pooled samples can be undertaken with acceptable loss of sensitivity, and advocate such an approach where test capacity is limited. We provide an approach to calculating the most efficient pool size. Given the current limitations of tests for SARS-CoV-2 infection, physical distancing and meticulous infection prevention and control will remain essential in institutions caring for vulnerable people.
\end{abstract}

\section{Keywords}

COVID-19, SARS Coronavirus 2, Infection Prevention and Control, Clinical Diagnostics, Epidemiology 


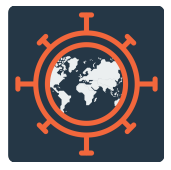

This article is included in the Emerging Diseases

and Outbreaks gateway.

collection.

Corresponding author: Tom A. Yates (t.yates@imperial.ac.uk)

Author roles: Yates TA: Conceptualization, Visualization, Writing - Original Draft Preparation, Writing - Review \& Editing; Cooke GS: Writing - Review \& Editing; MacPherson P: Visualization, Writing - Review \& Editing

Competing interests: No competing interests were disclosed.

Grant information: TAY is funded via an NIHR Academic Clinical Fellowship and acknowledges support from the NIHR Imperial Biomedical Research Centre. GSC is supported in part by the Biomedical Research Centre of Imperial College National Health Service (NHS) Trust and a National Institute for Health Research (NIHR) Research Professorship. PM is funded by the Wellcome Trust [206575]. The funders had no role in study design, data collection and analysis, decision to publish, or preparation of the manuscript.

Copyright: $\odot 2020$ Yates TA et al. This is an open access article distributed under the terms of the Creative Commons Attribution License, which permits unrestricted use, distribution, and reproduction in any medium, provided the original work is properly cited.

How to cite this article: Yates TA, Cooke GS and MacPherson P. Rational use of SARS-CoV-2 polymerase chain reaction tests within institutions caring for the vulnerable [version 1; peer review: 2 approved] F1000Research 2020, 9:671

https://doi.org/10.12688/f1000research.24872.1

First published: 02 Jul 2020, 9:671 https://doi.org/10.12688/f1000research.24872.1 


\section{Introduction}

Whilst there are significant limitations to the prognostic models that have been published to date ${ }^{1}$, the association between older age and death in SARS-CoV-2 infection is both clear and striking - the infection fatality rate for people in their 80 's has been estimated at $7.8 \%{ }^{2}$. Infection prevention and control (IPC) is therefore crucial within institutions that bring together many such individuals, such as hospitals and nursing or long-stay residential homes.

Here we discuss the use of polymerase chain reaction (PCR) tests within institutions caring for vulnerable individuals. We emphasise that consideration of test characteristics and the prior probability of SARS-CoV-2 infection is essential in making safe decisions based on PCR results. For example, decisions regarding the allocation of side rooms, whether patients can be cohorted in COVID or non-COVID spaces, and whether staff should be at work. We discuss the different uses of PCR tests, and consider which testing strategies should be prioritised. We explore how pooling samples prior to testing can result in a more efficient use of test capacity, and provide an optimised approach to selecting the number of samples to pool. Finally, we suggest how staff testing programmes for institutional care settings might be efficiently organised. We do not discuss disease surveillance or the role of testing for disease control in the wider community, but would point readers to recent modelling studies ${ }^{3,4}$. We only briefly discuss serological tests, as these are not yet in routine use. Our intended audience are those designing testing strategies in health and social care settings, as well as individuals in these settings acting on test results.

\section{Epidemiology and importance of transmission dynamics for institutional care settings}

High rates of SARS-CoV-2 infection with consequent morbidity and mortality have been observed in healthcare facilities and social care settings. Two infection prevalence surveys conducted in April and May 2020 in England found 1.33\% and 1.73\% of people working in 'patient-facing healthcare or resident-facing social care roles' had positive SARS-CoV-2 PCRs as compared with $0.22 \%$ and $0.38 \%$ of people not working in such roles ${ }^{5,6}$. Over much of this period, health and social care workers were one of the few groups permitted to leave their homes to work.

A transmission model parameterised to a typical hospital in the United Kingdom suggests that up to $20 \%$ of infections in patients and $89 \%$ of infections in healthcare workers may be a result of nosocomial transmission ${ }^{7}$. Consistent with that, SARS-CoV-2 infections in healthcare workers and staff have been observed to cluster on particular hospital wards ${ }^{8}$ and a large proportion of hospital staff working in particularly exposed roles, such as housekeepers $(34.5 \%)$ and those working in acute medicine $(33.3 \%)$, show serological evidence of having been infected during the initial weeks of the pandemic 9 .

Large transmission clusters of SARS-CoV-2 infections have been seen in hospitals and elderly care facilities, along with worker dormitories and ships ${ }^{10}$. In one detailed case report incorporating phylogenetic analysis, a single introduction of SARS-CoV-2 into a private hospital in South Africa led to 80 staff members and 39 patients becoming infected, with 15 patient deaths ${ }^{11}$. The consequences of transmission within the social care sector have, perhaps, been even more severe. In one well-described outbreak in a 'skilled nursing facility', 57/89 (64\%) of residents were infected, of whom fifteen died; a case fatality rate of $26 \%{ }^{12}$. Viral sequence data were consistent with two separate introductions of SARS-CoV-2 into the facility ${ }^{12}$.

A key feature of the epidemiology of SARS-CoV-2 transmission is that viral shedding begins 2-3 days prior to symptom onset ${ }^{13}$. A high proportion of transmission is thought to occur prior to symptom onset, estimated at $40-50 \%$ in studies from different settings ${ }^{13-16}$. Disease control strategies that rely on isolation once symptoms develop are, therefore, unlikely to be sufficient ${ }^{17}$.

Whilst a majority of those with SARS-CoV-2 infection do eventually develop symptoms, an appreciable prevalence of asymptomatic - rather than pre-symptomatic - infection has been described in hospital patients $(12.4 \%)^{18}$, nursing home residents $(3.9 \%)^{12}$ and healthcare workers $(0.5 \%)^{8}$. The contribution that these individuals make to transmission is less clear. The absence of cough and coryza might limit infectivity. Alternatively, a period of viral shedding without the social distancing and reductions in numbers of close contacts, which would have otherwise been prompted by symptom onset, might result in a greater number of secondary infections. Data suggest that pre-symptomatic transmission probably plays a more important role than asymptomatic transmission ${ }^{16}$. However, until this is better understood it would be prudent to consider both asymptomatic and pre-symptomatic people with positive SARSCoV-2 PCRs infectious, particularly given this group are initially clinically indistinguishable.

Clearly, the prevalence or prior probability of SARS-CoV-2 infection, among both individuals with and without symptoms, will vary over the course of the pandemic ${ }^{19}$ and with changes in control strategies.

\section{Test characteristics}

Viral load in the upper airway, the site most readily sampled, is thought to peak around the time of symptom onset ${ }^{13}$ and to be correlated with symptom severity ${ }^{20}$. An early analysis suggested that the sensitivity of PCR-based tests decreases with time since symptom onset, with only $67 \%$ of nasal and $47 \%$ of throat specimens expected to be PCR positive ten days after symptom onset ${ }^{21}$. In a small series of well-characterised patients with mild disease, test sensitivity was reported to be only $40 \%$ five days after symptom onset ${ }^{22}$. Later analysis, incorporating some of the same data, estimated that the sensitivity of the test was $62 \%$ on the day of symptom onset, $80 \%$ on day three after symptom onset, then declining to $33 \%$ by day 16 post symptom onset ${ }^{23}$. The sensitivity of a SARS-CoV-2 PCR on the day prior to symptom onset was estimated to be between $6 \%$ and $73 \%$, with the imprecision in the estimate reflecting the limited data available on pre-symptomatic patients ${ }^{23}$. Most of these studies did serial testing and required at least one positive PCR to consider a case confirmed - as a result they 
may have overestimated the sensitivity of the test. Some of the studies included in Kucirka et al. ${ }^{23}$ allowed probable cases, defined on clinical grounds, with a majority of these probable cases also having IgM or IgG antibodies against SARS-CoV-2.

Data on test specificity is less readily available. As well as the inherent performance of the test, specificity can be impacted by: clinicians swabbing the wrong patient or placing the wrong sticker on the specimen; RNA contamination at any stage in the process; and transcription errors when reporting the result. The extent to which these errors occur will be context specific and dependent on how robust local procedures are. Importantly, PCR based testing only gives a snapshot at one point in time. Individuals early in their infection may test negative then positive a few hours later ${ }^{23}$. As with all PCR based tests, poor sampling may result in false negatives.

Readily available formulae ${ }^{24}$ enable the probability of both false negative and false positive tests to be estimated under a range of assumptions about sensitivity, specificity, and the prevalence of the condition in the population being tested.

\section{Testing symptomatic staff in institutional care settings}

A major concern during the COVID-19 pandemic has been health systems' ability to cope with large numbers of acutely unwell individuals presenting to health facilities within a short period of time, overwhelming capacity. The availability of skilled clinicians is a major constraint on health system capacity and high rates of absenteeism have been reported at many centres ${ }^{25}$. Absent staff were either symptomatic, self isolating because a household contact was symptomatic, or not working as a result of medical conditions that would put them at risk of severe disease were they to acquire SARS-CoV-2. There has, thus, been great interest in SARS-CoV-2 PCR tests for symptomatic health and social care workers and their symptomatic household contacts.

Whilst reducing absenteeism is clearly important, a key concern when testing symptomatic individuals is ensuring that false negative results don't result in avoidable transmission of the virus among keyworkers and the vulnerable individuals they work with. Estimated false negative rates, under a range of plausible assumptions, are presented in Table 1.

As others have also noted ${ }^{21,23,26}$ high rates of false negative tests are seen when the prevalence of infection is high, and when the sensitivity of the test is low. The proportion of health and social care workers who have positive test results should be monitored with these data, ideally, disaggregated by symptoms. Where this proportion is high, there will be an unacceptable false negative rate and institutions should consider asking symptomatic staff to self isolate regardless of their test result. Examples of such risk stratification have been published ${ }^{8}$ but, in our view, should be kept under review, given the false negative rate will vary with the prevalence of infection in the population. It may be prudent to only test key workers shortly after symptom onset, when the sensitivity of the test is highest.

\begin{abstract}
Table 1. Estimated probability of a false negative SARS-CoV-2 PCR test under various assumptions about the true prevalence of infection and the sensitivity of the test ${ }^{24}$. All estimates assume a test specificity of $99.5 \%$.
\end{abstract}

\begin{tabular}{|l|l|l|l|}
\hline $\begin{array}{l}\text { True } \\
\text { prevalence } \\
\text { of infection }\end{array}$ & \multicolumn{3}{|l|}{$\begin{array}{l}\text { Probability of SARS-CoV-2 infection, } \\
\text { given negative PCR test }\end{array}$} \\
\hline & $\begin{array}{l}\text { 95\% test } \\
\text { sensitivity }\end{array}$ & $\begin{array}{l}\text { 70\% test } \\
\text { sensitivity }\end{array}$ & $\begin{array}{l}\text { 40\% test } \\
\text { sensitivity }\end{array}$ \\
\hline $90 \%$ & $31 \%$ & $73 \%$ & $84 \%$ \\
\hline $30 \%$ & $2.1 \%$ & $11 \%$ & $21 \%$ \\
\hline $5 \%$ & $0.26 \%$ & $1.6 \%$ & $3.1 \%$ \\
\hline
\end{tabular}

It is biologically plausible that staff with SARS-CoV-2 infection may be less infectious if they have a negative PCR. We know of no good data to support such an assertion and would urge caution, as negative results can be a result of poor sampling or virus being less readily detected in the upper respiratory tract than in samples taken from the lower airways ${ }^{27}$.

As others have also argued ${ }^{3}$, we feel it likely that any gains in terms of reduced absenteeism as a result of a programme of testing symptomatic staff may be modest. Staff with symptoms may be too unwell to work and, regardless, should not be working whilst symptomatic given other respiratory viruses can also be transmitted to patients. Testing symptomatic household contacts may be more impactful with, potentially, a lower pre-test probability, allowing greater confidence in a negative result ${ }^{3}$.

\section{Testing asymptomatic staff}

Arguably, a better use of tests, particularly if capacity is limited, would be systematic regular testing of asymptomatic staff, to drive down the prevalence of asymptomatic and pre-symptomatic infection within institutions caring for the vulnerable. One model suggests that weekly PCR testing of asymptomatic health and social care workers could reduce their contribution to incident infections by $25-33 \%$, over and above any reductions as a result of them self-isolating at onset of symptoms ${ }^{3}$. Given virus is likely only detectable 1-2 days prior to symptom onset ${ }^{13,23}$, one might expect more frequent testing to result in greater declines in transmission, particularly if results could be fed back promptly. A second model gave similar results regards the impact of weekly testing and suggested that daily testing of hospital staff could reduce healthcare worker to healthcare worker transmission by $65 \%$ and healthcare worker to patient transmission by $14 \%$.

Whilst, initially, a programme of testing asymptomatic staff might result in additional absenteeism, reductions in transmission may result in a favourable impact on staffing levels in the longer term $^{7}$. False positive tests would cause unnecessary absence from work, but no direct harm to vulnerable individuals.

The main barrier to regular asymptomatic testing is test capacity. For example, there are 1.5 million people working in 
adult social care in England ${ }^{28}$. Pooling samples prior to testing could make regular systematic large scale testing of key workers feasible. This is discussed in detail below.

The impact of asymptomatic staff testing could be increased by calibrating test frequency to the proportion of staff and patients testing positive and by offering enhanced support with other aspects of IPC to facilities with higher than expected rates of positive tests - this approach has been described in the hospital setting .

\section{Testing asymptomatic patients}

Following reports of high rates of asymptomatic infection in patients presenting to hospital with other issues ${ }^{18}$, many hospitals are now undertaking SARS-CoV-2 PCR tests on all new admissions, with the result used to distribute patients to 'COVID areas', 'non COVID areas' or side rooms. With potential for harm should people with SARS-CoV-2 infection and vulnerable people who have not yet been infected be placed in the same space, it is important to think critically about the positive and negative predictive value of the test in specific circumstances.

People with a positive PCR result and symptoms consistent with COVID-19, as well as asymptomatic individuals with a negative PCR test, pose fewer challenges when drafting infection control guidelines. Note, however, that the latter group may subsequently become PCR positive, either a result of nosocomial infection or because they were in their incubation period and PCR negative at presentation.

People with COVID-19 symptoms, or imaging and blood tests consistent with COVID, but a negative SARS-CoV-2 PCR are commonly seen in clinical practice ${ }^{29}$. Sometimes repeat PCR testing, particularly if a sample from the lower respiratory tract can be obtained, can confirm the diagnosis. Efforts should also be made to exclude alternative diagnoses, such as Pneumocystis jirovecii pneumonia, which can present in a similar manner.

The opposite phenomenon - a positive SARS-CoV-2 PCR in an asymptomatic person - can also occur. However, the possibility of this being an erroneous result is less well appreciated. In Table 2, are the probabilities of SARS-CoV-2 infection given a positive PCR that might be expected under various scenarios. False positive results will commonly be seen, particularly in settings and populations where the prevalence of infection is low.

Ideally, individuals with PCR test results that are not concordant with their symptoms would be cared for in side rooms. However, many institutions have a limited number of side rooms, meaning such a policy may not be sustainable. Requiring a second positive test in asymptomatic people with an initial positive PCR would seem prudent before moving them into a 'COVID area'.

Where individuals' infection status cannot be resolved and side rooms are not available, every effort should be taken to avoid placing individuals who would be particularly at risk of severe disease should they newly acquire infection in a space with individuals who may be infectious. Such a triage approach
Table 2. Estimated probability of SARS-CoV-2 infection given a positive PCR under various assumptions about the true prevalence of infection and the sensitivity of the test $^{24}$. All estimates assume a test specificity of $99.5 \%$.

\begin{tabular}{|l|l|l|l|}
\hline $\begin{array}{l}\text { True } \\
\text { prevalence of } \\
\text { infection }\end{array}$ & \multicolumn{3}{|l|}{$\begin{array}{l}\text { Probability of SARS-CoV-2 infection, } \\
\text { given positive PCR test }\end{array}$} \\
\hline & $\begin{array}{l}\text { 95\% test } \\
\text { sensitivity }\end{array}$ & $\begin{array}{l}\mathbf{7 0 \%} \text { test } \\
\text { sensitivity }\end{array}$ & $\begin{array}{l}\mathbf{4 0 \%} \text { test } \\
\text { sensitivity }\end{array}$ \\
\hline $15 \%$ & $97 \%$ & $96 \%$ & $93 \%$ \\
\hline $5 \%$ & $91 \%$ & $88 \%$ & $81 \%$ \\
\hline $1 \%$ & $66 \%$ & $59 \%$ & $45 \%$ \\
\hline
\end{tabular}

has been piloted at one hospital - in this case with the triage decision taken prior to the PCR result being available ${ }^{29}$. The performance of any such algorithm will vary with the background prevalence of SARS-CoV-2 infection. If vulnerable and potentially uninfected individuals must be moved out of side rooms, impeccable infection control measures should be adopted in the spaces to which they are moved, perhaps supported by better staff-to-client ratios. If possible, such spaces should be closed to new admissions to prevent these individuals being exposed to new people who may be infectious.

\section{Pooling specimens prior to analysis}

One way that testing capacity might be expanded is by pooling specimens prior to analysis. A recent report suggests that pooling of samples either prior to or following RNA extraction can allow multiple samples to be tested in a single PCR reaction without significant loss of sensitivity ${ }^{30}$.

The authors reported a 1.24 increase in the PCR cycle threshold with every two-fold dilution of the sample - i.e. going from one to two, or two to four samples. In their hands, pooling 32 samples following RNA extraction reduced the sensitivity of the test by $10 \%$. They suggest that some of this loss of sensitivity might be overcome by running the PCR for a few additional cycles. Any loss of specificity as a result of additional PCR cycles would not be expected to impact patient management, as individual samples from pools that tested positive would then be retested using one PCR reaction per sample.

The probability that, in any pool of specimens, at least one will test positive is one minus the probability that all will test negative. Here, the prevalence is the prevalence of positive tests, were each to be tested in a separate PCR run, rather than the true prevalence of infection.

\section{Probability $\geq 1$ positive $=1-(1-\text { prevalence })^{\wedge}$ number of tests}

The expected number of pools that test positive would be the product of this probability, the number of pools, and the sensitivity of the pooled approach, as compared with one PCR per sample. The number of infections missed, as compared with a one PCR reaction per sample strategy, would approximate 
the product of the true prevalence of infection, the loss of sensitivity as compared with a one PCR reaction per sample strategy, and the number of individuals tested.

The most efficient pool size depends on the prevalence (Figure 1). The scenario illustrated in this figure assumes a health or social care workforce of 10,000 individuals and the number of PCR reactions calculated assumes individual PCRs are then performed on each sample in pools that test positive. Again, prevalence is the prevalence of positive tests, were each to be tested in a separate PCR run, rather than the true prevalence of infection.

The most efficient pool size is readily calculable, and the prevalence of positive tests should be monitored to ensure the optimal pool size is being used. At higher prevalence, larger pools are less efficient, as the high proportion of pools testing positive limits the number of tests saved.

\section{Box 1. Template staff testing strategy for institutions caring for} vulnerable people

Briefing for staff on the planned testing strategy

Sampling frame drawn up for each ward/facility, to include all medical, nursing, allied health professionals, and domestic staff with a regular presence in the institution

Collect mobile phone numbers for each staff member, and issue each with a patient number, if they do not already have one

Collate rotas and prepare two swabs for each staff member per week, pre-labelled and in lysis buffer (likely to equate to approximately alternate day testing, assuming some rest days)

Allocate these to packets that are delivered to wards/facilities ahead of time with one packet for each morning handover

After morning handover, self nose and throat swabs by outgoing night staff and incoming day staff

Testing register to include space to note whether any staff have left or joined - swabs for new staff could be added to subsequent test packets

Specimens collected after handover to allow RT-PCR for SARS CoV-2 on a mid morning PCR run - this may need a dedicated courier collection

Positive results called out before $5 \mathrm{pm}$, to ensure incoming night staff don't come on shift (if unable to contact staff member, message to be left with nurse in charge)

Individual letters, emails or text messages to each staff member, containing test results, plus reliable and timely communication of positive results to local infection control and health protection teams

Clear messaging to ensure symptomatic staff do not wait for their test result before leaving work, and that individuals developing symptoms self isolate regardless of whether they have had a recent negative test

Dedicated mobile phone and email address for any queries

\section{Organisational considerations for asymptomatic staff testing}

In designing a testing strategy for asymptomatic staff, there will be trade offs. For example, between a one PCR reaction per sample strategy and a pooled testing strategy. The former would be expected to result in the fastest results if there was plenty of capacity, whereas the latter would make most efficient use of limited capacity but add a few hours to the turn around time.

Duration of infectiousness prior to symptom onset is thought to be between 2-3 days ${ }^{13}$ with limited PCR sensitivity expected more than two days prior to symptom onset ${ }^{23}$. We would, therefore, advocate an asymptomatic staff testing strategy that prioritises frequent testing and prompt reporting of results over marginal improvements in test sensitivity.

Having nose and throat swabs taken by individuals trained to do this might result in better sampling, increasing test sensitivity, but would place limits on how quickly testing could be scaled up. Of note, 1533 swabs taken by symptomatic staff at a hospital in England were all positive for RNAaseP, a human nucleic acid used as a sample quality control. This does not mean that sampling was perfect, but suggests that the swabs did all make contact with some human mucosa ${ }^{31}$. In a smaller study, good agreement was seen between PCR results using self swabs and swabs taken by a trained professional ${ }^{32}$.

There is some data to suggest that saliva may represent a viable alternative upper respiratory tract sample to nose and throat swabs for SARS-CoV-2 PCR. Of the two studies published to date, one found saliva had slightly better sensitivity than nose and throat swabs ${ }^{33}$, and one found the opposite ${ }^{34}$. As one might expect, given saliva samples are easier to collect, greater consistency was seen between PCR results on repeat saliva samples than with repeat nose and throat swabs ${ }^{33}$. Where selfsampling is planned, laboratories may wish to validate PCR assays using saliva.

A suggestion as to how a mass testing strategy for staff might be organised is outlined in Box 1. Other choices may be more appropriate, depending on local circumstances. For example, care homes may wish to include regular visitors in their sampling frame and to mandate a recent negative PCR prior to visiting. Facilities using agency staff may wish to mandate a recent negative PCR prior to any shifts. Testing intensity could be calibrated to local epidemiology and testing capacity ${ }^{8}$, with a move to daily testing when capacity is available or if high rates of asymptomatic infection or disease are seen within facilities. Testing programmes should be developed in close collaboration with clinicians, infection control teams, health protection teams and the workers that are to be tested.

Duration of self-isolation should be compliant with local guidelines, which are likely to evolve as our understanding of duration of infectiousness changes. Unless staff are immunocompromised, mandating negative PCRs before return to work would, in our view, be over cautious given PCR tests may detect non viable virus. Immunocompromised people should probably not be undertaking patient facing duties currently. In the absence of good data on duration of immunity following natural infection, pragmatic decisions may need to be taken locally about whether 


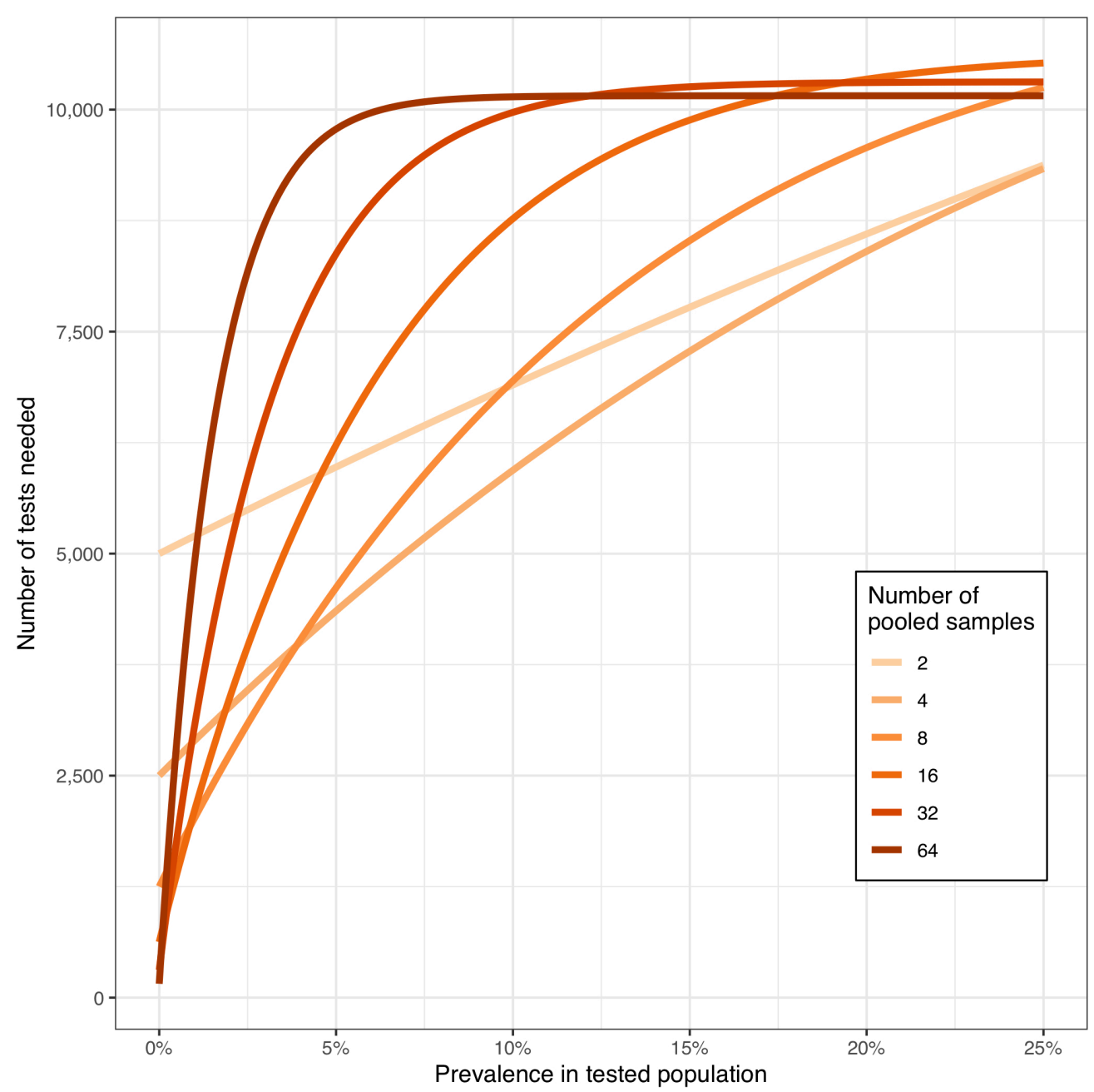

Figure 1. Number of PCR tests required as a function of pool size at different prevalences in a hypothetical population of 10,000 individuals.

individuals who have had a positive SARS-CoV-2 PCR continue to be included in asymptomatic staff testing programmes.

\section{Serological tests}

If and when we develop an understanding of the immunological correlates of protection against SARS-CoV-2 infection and or COVID-19, and have validated assays to measure this, they may compliment PCR based testing. For example, if we had a serological assay that could identify individuals with protective immunity to infection or disease, positive serology could allow someone to be safely moved out of a side room and into a 'COVID area'. Such a test could also identify individuals that would no longer need to be included in asymptomatic staff testing programmes. For both of these purposes, a highly specific test would be needed.

\section{Key messages}

- Correct interpretation of SARS-CoV-2 PCR tests depends on the sensitivity of the test and prevalence of infection in the population being tested.
- Test sensitivity declines with time since onset of symptoms.

- The prevalence of infection will vary between groups, with the symptoms people report, and over the course of the pandemic - monitoring the proportion of PCR tests that are positive can give an estimate of the prior probability of a positive test.

- Where prevalence of infection is high, or more than three days have elapsed since symptom onset, a negative PCR may not reliably exclude SARS-CoV-2 infection and, in this situation, symptomatic individuals should isolate whatever their PCR result.

- Where the prevalence of infection is low, false positive PCR tests may be commonly seen - it is prudent to obtain a second positive test before placing vulnerable individuals with a single positive test in a space containing other people with SARS-CoV-2 infection. 
- Using test capacity to reduce the prevalence of SARS-CoV-2 infection in institutions accommodating vulnerable individual should be a priority, with models predicting a substantial impact on transmission.

- A significant proportion of SARS-CoV-2 transmission occurs prior to symptom onset and there is a window of 1-2 days prior to symptom onset when a PCR test might be positive - regular testing of asymptomatic individuals and the prompt reporting of results are therefore needed.
- Test capacity can be substantially increased by pooling samples prior to testing - the most efficient pool size will depend on the prevalence of infection and is readily calculated.

- Physical distancing and meticulous infection prevention and control will remain essential in institutions caring for vulnerable people.

\section{Data availability}

No data are associated with this article.
1. Wynants L, Van Calster B, Collins GS, et al.: Prediction models for diagnosis and prognosis of covid-19 infection: Systematic review and critical appraisal. BMJ. 2020; 369: $\mathrm{m} 1328$

PubMed Abstract | Publisher Full Text | Free Full Text

2. Verity R, Okell LC, Dorigatti I, et al:: Estimates of the severity of coronavirus disease 2019: a model-based analysis. Lancet Infect Dis. 2020; 20(6): 669-677. PubMed Abstract | Publisher Full Text | Free Full Text

3. Grassly NC, Pons-Salort M, Parker EPK, et al.: Role of testing in COVID-19 control. London; 2020.

Reference Source

4. Kucharski AJ, Klepac P, Conlan A, et al.: Effectiveness of isolation, testing, contact tracing, and physical distancing on reducing transmission of SARS-CoV-2 in different settings: a mathematical modelling study. Lancet Infect Dis. 2020; S1473-3099(20)30457-6.

PubMed Abstract | Publisher Full Text

5. Office for National Statistics: Coronavirus (COVID-19) Infection Survey pilot: England, 14 May 2020. 2020.

Reference Source

6. Office for National Statistics: Coronavirus (COVID-19) Infection Survey pilot: 28 May 2020. [Internet]. 2020 [cited 2020 May 29]. Reference Source

7. Evans S, Agnew E, Vynnycky E, et al.: The impact of testing and infection prevention and control strategies on within-hospital transmission dynamics of COVID-19 in English hospitals. medRxiv. 2020; 2020.05.12.20095562. Publisher Full Text

8. Rivett L, Sridhar S, Sparkes D, et al:: Screening of healthcare workers for SARS CoV-2 highlights the role of asymptomatic carriage in COVID-19 transmission. Elife. 2020; 9: e58728.

PubMed Abstract | Publisher Full Text

9. Shields AM, Faustini SE, Perez-Toledo M, et al:: SARS-CoV-2 seroconversion in health care workers. medRxiv. 2020; 2020.05.18.20105197.

Publisher Full Text

10. Leclerc QJ, Fuller NM, Knight LE, et al.: What settings have been linked to SARS-CoV-2 transmission clusters? [version 1; peer review: 1 approved with reservations] Wellcome Open Res. 2020; 5: 83. Publisher Full Text

11. Lessells R, Moosa Y, de Oliveira T: Report into a nosocomial outbreak of coronavirus disease 2019 (COVID-19) at Netcare St Augustine's Hospital. [Internet]. Durban; 2020.

Reference Source

12. Arons MM, Hatfield KM, Reddy SC, et al:: Presymptomatic SARS-CoV-2 Infections and Transmission in a Skilled Nursing Facility. N Engl J Med. 2020; 382(22): 1-10.

PubMed Abstract | Publisher Full Text | Free Full Text

13. He X, Lau EH, Wu P, et al.: Temporal dynamics in viral shedding and transmissibility of COVID-19. Nat Med. 2020; 26: 672-675. Publisher Full Text

14. Ganyani T, Kremer C, Chen D, et al: Estimating the generation interval for coronavirus disease (COVID-19) based on symptom onset data, March 2020. Euro Surveill. 2020; 25(17): 2000257.

PubMed Abstract | Publisher Full Text | Free Full Text

15. Liu Y, Funk S, Flasche $S$, et al:: The contribution of pre-symptomatic infection to the transmission dynamics of COVID-2019 [version 1; peer review: 1 approved]. Wellcome Open Res. 2020; 5: 58. Publisher Full Text

16. Ferretti L, Wymant C, Kendall M, et al:: Quantifying SARS-CoV-2 transmission suggests epidemic control with digital contact tracing. Science. 2020; 368(6491): eabb6936.

PubMed Abstract | Publisher Full Text | Free Full Text
17. Fraser C, Riley S, Anderson RM, et al.: Factors that make an infectious disease outbreak controllable. Proc Natl Acad Sci U S A. 2004; 101(16): 6146-51. PubMed Abstract | Publisher Full Text | Free Full Text

18. Sutton D, Fuchs $K$, D'Alton M, et al.: Universal Screening for SARS-CoV-2 in Women Admitted for Delivery. N Engl J Med. 2020; 382: 2163-2164. Publisher Full Text

19. Treibel TA, Manisty C, Burton M, et al:: COVID-19: PCR screening of asymptomatic health- care workers at London hospital. Lancet. 2020; 395(10237): 1608-1610.

PubMed Abstract | Publisher Full Text | Free Full Text

20. Liu Y, Yan LM, Wan L, et al.: Viral dynamics in mild and severe cases of CoVID-19. Lancet Infect Dis. 2020; 20(6): 656-657. PubMed Abstract | Publisher Full Text | Free Full Text

21. Wikramaratna $P$, Paton RS, Ghafari M, et al.: Estimating false-negative detection rate of SARS-CoV-2 by RT-PCR. medRxiv. 2020; 2020.04.05.20053355. Publisher Full Text

22. Wölfel R, Corman VM, Guggemos W, et al:: Virological assessment of hospitalized patients with COVID-2019. Nature. 2020; 581(7809): 465-469. PubMed Abstract | Publisher Full Text

23. Kucirka LM, Lauer SA, Laeyendecker O, et al: Variation in False-Negative Rate of Reverse Transcriptase Polymerase Chain Reaction-Based SARS-CoV-2 Tests by Time Since Exposure. Ann Intern Med. 2020; M20-1495. PubMed Abstract | Publisher Full Text | Free Full Text

24. Morgan D: Medical Test Calculator. [Internet]. 2020. Reference Source

25. Royal College of Physicians: COVID-19 and its impact on NHS workforce. 2020 Reference Source

26. Watson J, Whiting PF, Brush JE: Interpreting a covid-19 test result. BMJ. 2020; 369: $\mathrm{m} 1808$

PubMed Abstract

27. Wang D, Hu B, Hu C, et al:: Clinical Characteristics of 138 Hospitalized Patients with 2019 Novel Coronavirus-Infected Pneumonia in Wuhan, China. JAMA. 2020; 323(11): 1061-9.

PubMed Abstract | Publisher Full Text | Free Full Text

28. Griffiths D, Fenton W, Polzin G, et al.: The state of the adult social care sector and workforce in England. [Internet]. Leeds. 2019. Reference Source

29. Patterson B, Marks M, Martinez-garcia G, et al:: A Novel Cohorting and Isolation Strategy for Suspected COVID-19 Cases during a Pandemic. J Hosp Infact. 2020; S0195-6701(20)30275-9

PubMed Abstract | Publisher Full Text | Free Full Text

30. Yelin I, Aharony N, Tamar ES, et al.: Evaluation of COVID-19 RT-qPCR test in multi-sample pools Idan. Clin Infact Dis. 2020; ciaa531. PubMed Abstract | Publisher Full Text | Free Full Text

31. Keeley AJ, Evans C, Colton H, et al:: Roll-out of SARS-CoV-2 testing for healthcare workers at a large NHS Foundation Trust in the United. Euro Surveill. 2020; 25(14): 2000433.

PubMed Abstract | Publisher Full Text | Free Full Text

32. Wehrhahn MC, Robson J, Brown S, et al.: Self-collection: an appropriate alternative during the SARS-CoV-2 pandemic. J Clin Virol. 2020. 128: 104417. PublMed Abstract | Publisher Full Text | Free Full Text

33. Wyllie AL, Fournier J, Casanovas-Massana A, et al: Saliva is more sensitive for SARS-CoV-2 detection in COVID-19 patients than nasopharyngeal swabs. medRxiv. 2020; 2020.04.16.20067835. Publisher Full Text

34. Williams $\mathrm{E}$, Bond $\mathrm{K}$, Zhang $\mathrm{B}$, et al: Saliva as a non-invasive specimen for detection of SARS-CoV-2. J Clin Microbiol. 2020; JCM.00776-20. PubMed Abstract | Publisher Full Text 


\title{
Open Peer Review
}

\section{Current Peer Review Status:}

\section{Version 1}

Reviewer Report 11 September 2020

https://doi.org/10.5256/f1000research.27443.r69772

(C) 2020 Jeffery $\mathrm{K}$ et al. This is an open access peer review report distributed under the terms of the Creative Commons Attribution License, which permits unrestricted use, distribution, and reproduction in any medium, provided the original work is properly cited.

\author{
Sheila Lumley \\ ${ }^{1}$ Nuffield Department of Medicine, University of Oxford, Oxford, UK \\ 2 Oxford University Hospital NHS Foundation Trust, Oxford, UK
}

\section{Katie Jeffery}

Department of Infectious Diseases and Microbiology, John Radcliffe Hospital, Oxford University Hospitals NHS Foundation Trust, Oxford, UK

Yates et al. discuss the current and highly topical issue of infection prevention control in institutional care facilities, focusing on the benefits of high frequency surveillance of asymptomatic and pre-symptomatic individuals, on absenteeism and infection prevention control. The article covers a range of important considerations, including sampling strategies (selfswabbing and saliva collection), testing strategies in particular pooling of samples, result interpretation given different population prevalence, and organisational considerations for implementing such a strategy. This article discusses how the pooling of samples might overcome the current capacity issues when implementing a high frequency mass screening strategy, given current constraints with NHS testing capacity to deliver screening programmes for large numbers of staff or patients.

A number of papers on screening programmes in healthcare workers show high rates of nosocomial acquisition in staff who were 'COVID-facing'. Much of this occurred in the early part of the pandemic in the UK before the introduction of universal PPE, with individuals looking after elderly patients or those with atypical COVID-19 presentations in the early part of the pandemic, leading to mode of acquisition in line with the reference quoted (89\% of infections in healthcare workers may be a result of nosocomial transmission). However, following the introduction of universal PPE, the literature also documents high rates of staff to staff transmission in non-patient facing areas likely due to a failure of staff to socially distance away from clinical areas. In a study of over 10000 healthcare workers in an acute Trust the greatest risk of staff infection was associated with having a Covid-19 infected contact in the household (eLife 2020;9:e60675 DOI: 10.7554/eLife.60675) ${ }^{1}$. Most Healthcare settings are now seeing lower rates of nosocomial and staff to staff transmission, given universal mask wearing in the UK. The authors' intended audience is those designing testing strategies in health and social care settings, however, a 
number of the strategies discussed also have broader application for mass screening in lower prevalence settings such as workplaces and educational facilities. Expanding local/regional access to testing, testing capacity, improving turnaround time, maintaining analytic performance appropriate for the setting and population prevalence will contribute importantly to the current national effort to curb the pandemic.

Comments to address:

Turnaround times - a key factor in high frequency testing, including pre-shift testing, is short turnaround times. How does a pooling strategy effect this - it seems unlikely that the necessary turnaround times (i.e. results before returning for the next shift) could be met if samples are being analysed in a laboratory setting and repeat testing of individual samples from a positive pool is required. See: https://www.ncbi.nlm.nih.gov/pmc/articles/PMC7325181/. ${ }^{2}$ Other assay formats could be considered such as point-of-care nucleic acid LAMP and rapid antigen tests - but given reduced sensitivity in comparison with laboratory based PCR assays modelling with respect to population prevalence would need to be carefully considered.

Referencing: The COVID literature is expanding at an exponential rate. As with much of current COVID literature, many papers already exist on similar topics, the context of this opinion article within the current literature isn't clear in places, with some citations lacking. This article would benefit from a more thorough literature review in the following areas:

1. Sample pooling is already used in a number of settings, with CDC guidance on the topic ( https://www.cdc.gov/coronavirus/2019-ncov/lab/pooling-procedures.html). The widespread use isn't represented in this paper, with only 1 reference. There are a number of other papers discussing pooling samples, more accurate representation of current literature is needed.

2. Testing of saliva - there are multiple recent papers on this topic.

Please clarify this paragraph: '...high rates of false negative tests are seen when the prevalence of infection is high, and when the sensitivity of the test is low. The proportion of health and social care workers who have positive test results should be monitored with these data, ideally, disaggregated by symptoms. Where this proportion is high, there will be an unacceptable false negative rate and institutions should consider asking symptomatic staff to self isolate regardless of their test result.' - when what proportion is high? The proportion of symptomatic vs asymptomatic? Proportion testing positive?

Serology: "Such a test could also identify individuals that would no longer need to be included in asymptomatic staff testing programmes" - this assumes that we know whether these individuals can be re-infected and still shed/transmit virus. The SIREN project (SARS-CoV-2 Immunity \& REinfection EvaluatioN) includes those previously infected so we can gain a better understanding of what having evidence of previous exposure means with respect to re-infection.

The article would benefit from a discussion of the information technology constraints - these are huge. Patient/sample identification at the point of test, sample labelling with barcodes allowing easier run-through processes in diagnostic laboratories, IT to support pooling/separation of pools, result notification to allow responses on an individual level and for contact tracing.

Conclusion 
At the time of writing this review (September 2020) the UK government is promising mass coronavirus testing ('operation Moonshot') but the technology to deliver this does not yet exist. It is vitally important to consider how mass-surveillance testing could be rolled out in institutional care settings. The pace at which testing is required to move forwards is unprecedented compared with anything we have seen before, and this opinion piece with its careful consideration of testing utility at different levels of population prevalence and assay sensitivity will be a useful article.

\section{References}

1. Eyre DW, Lumley SF, O'Donnell D, Campbell M, et al.: Differential occupational risks to healthcare workers from SARS-CoV-2 observed during a prospective observational study.Elife.

2020; 9. PubMed Abstract | Publisher Full Text

2. Larremore D, Wilder B, Lester E, Shehata $S$, et al.: Test sensitivity is secondary to frequency and turnaround time for COVID-19 surveillance. medRxiv. 2020. Publisher Full Text

Is the topic of the opinion article discussed accurately in the context of the current literature?

Partly

\section{Are all factual statements correct and adequately supported by citations?} Yes

Are arguments sufficiently supported by evidence from the published literature? Partly

Are the conclusions drawn balanced and justified on the basis of the presented arguments? Yes

Competing Interests: No competing interests were disclosed.

Reviewer Expertise: COVID: Asymptomatic healthcare worker surveillance/testing/contact tracing, laboratory testing, assay evaluation, infection prevention and control

We confirm that we have read this submission and believe that we have an appropriate level of expertise to confirm that it is of an acceptable scientific standard.

Reviewer Report 20 July 2020

https://doi.org/10.5256/f1000research.27443.r66235

(C) 2020 Agoti C. This is an open access peer review report distributed under the terms of the Creative Commons Attribution License, which permits unrestricted use, distribution, and reproduction in any medium, provided the original work is properly cited.

\section{Charles Agoti}

${ }^{1}$ Epidemiology and Demography Department, Kenya Medical Research Institute, Kilifi, Kenya 2 
Department of Public Health, School of Health and Human Sciences, Pwani University, Kilifi, Kenya

Yates et al. vividly discuss utility and inherent limitations of current PCR testing of SARS-CoV-2 in institutions caring for the elderly and vulnerable adults. They begin by summarizing the SARS-CoV2 epidemiology in this key population. The laboratory testing practical considerations and implications they highlight are very useful for both their specific target audience and the general scientific community to keep vulnerable populations safe. The messages of importance of quick sample-to-answer turnaround time, frequent testing to identify asymptomatic and presymptomatic infections, that PCR is not $100 \%$ accurate and that testing resources can be conserved by pooled testing are very important in these times. They provide suggestions on optimizing staff testing in care homes to monitor any SARS-CoV-2 infection introduction using available resources sparingly. I have three minor suggestions for the authors to consider.

1. Comment on if PCR cycle threshold ( $\mathrm{Ct}$ ) value is a useful indicator in evaluating the infectiousness of both staff and those under care and how this can be incorporated in making decisions in a care facility and in recognizing potential false positives?

2. Comment on whether sensitivity and specificity differ between PCR assays and how this can impact SARS-CoV-2 results in care homes. Any recommendations here? There are hundreds of commercial PCR assays available for SARS-CoV-2 testing. Some of the available PCR test check for multiple SARS-CoV-2 genes concurrently and include an internal control.

3. Comment on how/whether PCR tests can be used to identify contamination in care homes e.g. environmental swabbing, wastewater/sewage monitoring. Is this a viable strategy to monitor introduction or ongoing shedding/transmission?

Is the topic of the opinion article discussed accurately in the context of the current literature?

Yes

Are all factual statements correct and adequately supported by citations?

Yes

Are arguments sufficiently supported by evidence from the published literature? Yes

Are the conclusions drawn balanced and justified on the basis of the presented arguments? Yes

Competing Interests: No competing interests were disclosed.

Reviewer Expertise: Molecular diagnostics/viral genomics/Respiratory/Enteric virus transmission in community settings.

I confirm that I have read this submission and believe that I have an appropriate level of expertise to confirm that it is of an acceptable scientific standard. 
The benefits of publishing with F1000Research:

- Your article is published within days, with no editorial bias

- You can publish traditional articles, null/negative results, case reports, data notes and more

- The peer review process is transparent and collaborative

- Your article is indexed in PubMed after passing peer review

- Dedicated customer support at every stage

For pre-submission enquiries, contact research@f1000.com 